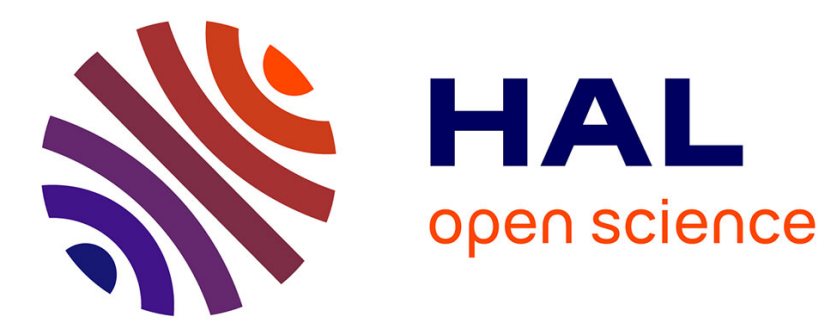

\title{
Dual operator algebras with normal virtual h-diagonal.
} Jean Roydor

\section{To cite this version:}

Jean Roydor. Dual operator algebras with normal virtual h-diagonal.. Integral Equations Operator Theory, 2012, Integral Equations Operator Theory 73 (2012), no. 3, 365-382. hal-01016331

\section{HAL Id: hal-01016331 \\ https://hal.science/hal-01016331}

Submitted on 30 Jun 2014

HAL is a multi-disciplinary open access archive for the deposit and dissemination of scientific research documents, whether they are published or not. The documents may come from teaching and research institutions in France or abroad, or from public or private research centers.
L'archive ouverte pluridisciplinaire HAL, est destinée au dépôt et à la diffusion de documents scientifiques de niveau recherche, publiés ou non, émanant des établissements d'enseignement et de recherche français ou étrangers, des laboratoires publics ou privés. 


\title{
DUAL OPERATOR ALGEBRAS WITH NORMAL VIRTUAL $h$-DIAGONAL
}

\author{
JEAN ROYDOR
}

\begin{abstract}
We study the class of dual operator algebras admitting a normal virtual $h$-diagonal (i.e. a diagonal in the normal Haagerup tensor product), this property can be seen as a dual operator space version of amenability. After giving several characterizations of these algebras, we show this class is stable under algebraic perturbations and cb-isomorphisms with small bound. We also prove some perturbation results for the Kadison-Kastler metric.
\end{abstract}

\section{INTRODUCTION}

In this paper, we are interested in a special class of dual operator algebras. Concretely a dual operator algebra is a $w^{*}$-closed subalgebra of a certain $\mathbb{B}(H)$, the von Neumann algebra of all bounded operators on a Hilbert space $H$ (see Section 2.7 in [2] for details on dual operator algebras), here we assume all our algebras to be unital. We consider the class of all dual operator algebras admitting a normal virtual $h$ diagonal in the sense of [2] Paragraph 7.4.8. Let us recall this notion (for background on completely bounded maps, operator space theory and non-selfadjoint algebra theory, the reader is referred to [2], [9], [14] and [16]). In the following $\otimes_{\sigma h}$ denotes the normal Haagerup tensor product, see Paragraph 1.6.8 of [2]. Let $\mathcal{M}$ be a unital dual operator algebra. The $\mathcal{M}$-bimodule structure of $\mathcal{M} \otimes_{\sigma h} \mathcal{M}$ is determined by the actions:

$$
a \cdot(c \otimes d) \cdot b=a c \otimes d b .
$$

We denote

$$
\mathrm{m}_{\mathcal{M}}: \mathcal{M} \otimes_{\sigma h} \mathcal{M} \rightarrow \mathcal{M}
$$

the completely contractive $w^{*}$-continuous linear map induced by the multiplication. A normal virtual $h$-diagonal for $\mathcal{M}$ is an element $u \in$ $\mathcal{M} \otimes_{\sigma h} \mathcal{M}$ satisfying:

(C1) for any $m \in \mathcal{M}, m \cdot u=u \cdot m$,

2000 Mathematics Subject Classification. 47L65, 47L55.

The author is supported by JSPS. 
In this paper we call such an element $\sigma h$-diagonal. Note that condition (C2) implies that the norm of a $\sigma h$-diagonal is always greater or equal to 1 (because $\mathrm{m}_{\mathcal{M}}$ is contractive). When a dual operator algebra $\mathcal{M}$ admits a $\sigma$-diagonal, $\mathcal{M}$ is said $\sigma$-amenable.

This condition can be thought as an amenability condition (it is an analog of virtual diagonal) in the category of dual operator algebras. Therefore we will try to transfer properties of amenable Banach algebras to $\sigma h$-amenable dual operator algebras. Especially we are interested in stability properties of this class of algebras under different type of perturbations: algebraic perturbations (i.e. neighboring multiplications), cb-isomorphisms and perturbation in the sense of KadisonKastler. Note also that admitting a normal virtual $h$-diagonal is a weaker condition than admitting a normal virtual diagonal (see remark 2.1 in [19]), hence in view of Conjecture 1 in [19], it is interesting to understand the class of $\sigma h$-amenable dual operator algebras.

In Section 2, we give different characterizations of $\sigma h$-amenability. Recall that the initial definition of amenability for Banach algebras is in terms of vanishing of the first Hochschild cohomology group on dual Banach modules. For dual Banach algebras, two analogs of amenability have already been considered: one in terms of normal virtual diagonal and another one in terms of vanishing cohomology on normal dual Banach bimodules (i.e. Connes-amenability, see [20]). The problem is that, unlike the Banach algebra case, this two notions do not coincide for dual Banach algebras, see [21]. This phenomenon can not happen for dual operator algebras; more precisely, $\sigma h$-amenability is equivalent to vanishing of the first Hochschild cohomology group relatively to the normal completely bounded cohomology, see Proposition 2.3. This actually relies on the associativity of the normal haagerup tensor product. The idea of considering the normal completely bounded cohomology is inspired from [23] (see also [15]). Furthermore, we also characterize $\sigma h$-amenability in terms of neighboring representations. The link between geometry of representations and amenability is known to Banach algebraists (see [20]). Finally as a direct application of Gifford's work, we describe $w^{*}$-closed ideals of $\sigma h$-amenable dual operator algebras (this description will be used in Proposition 3.13).

In Section 3, we study the stability properties of the class of $\sigma h$ amenable dual operator algebras. We first notice that $\sigma h$-amenability is stable under algebraic perturbations see Proposition 3.4. This result is an analog of Theorem 3 [18], but here we give a proof using [19], so that we can give an explicit bound in function of the norm 
of $\sigma h$-diagonal. Further, by an ultraproduct argument, we also prove that the class of $\sigma h$-amenable operator algebras is stable under unital $w^{*}$-continuous cb-isomorphisms with small bound (see Theorem 3.6). The objective of the second part of Section 3 is to prove perturbation results for the Kadison-Kastler metric in the continuation of [19]. Perturbations for various classes of non-selfadjoint algebras have already been considered (see e.g. [7], [17] and [13]). But here for our results, two hypothesis are needed: a complementation hypothesis (i.e. existence of a projection) and an amenability hypothesis (i.e. existence of a normal virtual $h$-diagonal or a normal virtual diagonal). In [19], we could prove a parametrization of Theorem 4.1 [5] in function of the norm of the normal virtual diagonal (but we kept a completely contractive projection). We use here the term 'parametrization' because a dual operator algebra which admits a normal virtual $h$-diagonal of norm one is actually selfadjoint. The same phenomenon occurs for the complementation hypothesis, more precisely a unital operator algebra which is the range of completely contractive projection is necessarily selfadjoint. Hence we could imagine to incorporate the cb-norm of the projection as an extra parameter, Theorem 4.1 [5] is almost the special case of Theorem 3.9 corresponding to cb-norm of the projection equals one. The only problem is that we need to assume this projection to be $w^{*}$-continuous. The only missing ingredient in order to find a 'full parametrization' of Theorem 4.1 [5] is an analog of Tomiyama's decomposition (into normal/singular parts, see [24]) for $\sigma h$-amenable (non-selfadjoint) dual operator algebras. We prove such a decomposition in the very special case when a dual operator algebra admits a virtual $h$-diagonal in the sense of [2]. As a peripheral result, we also show that a cb-complemented $\sigma h$-amenable dual operator algebra which is close enough to a von Neumann algebra is similar to it (see Theorem 3.12).

\section{Characterizations And Permanence Property}

In this section, we give two characterizations of unital dual operator algebras admitting a $\sigma h$-diagonal (see Corollary 2.7 below). The first one involves the normal completely bounded Hochschild cohomology of operator algebras. The second characterization is in terms of neighbouring representations.

Definition 2.1. Let $\mathcal{M}$ be a unital dual operator algebra and $\mathcal{X}$ be a dual operator space which is a $\mathcal{M}$-module. Then, $\mathcal{X}$ is called a normal operator $\mathcal{M}$-module if the bilinear map

$$
(m, x) \in \mathcal{M} \times \mathcal{X} \mapsto m \cdot x \in \mathcal{X}
$$


induced by the action of $\mathcal{M}$ on $\mathcal{X}$ is completely contractive and the map $m \in \mathcal{M} \mapsto m \cdot x \in \mathcal{X}$ is $w^{*}$-continuous, for every $x \in \mathcal{X}$.

The bimodule version of this definition is obvious (see Paragraph 3.8 .2 in [2] for more details).

One can be surprised that, in the previous definition, the $w^{*}$-continuity of the map $x \in \mathcal{X} \mapsto m \cdot x \in \mathcal{X}$ is not required. Actually this $w^{*}$ continuity is automatic from Corollary 4.10 in [3]. Consequently, from the universal property of the normal Haagerup tensor product, $\mathcal{X}$ is a normal operator $\mathcal{M}$-module if and only if

$$
m \otimes x \in \mathcal{M} \otimes \mathcal{X} \mapsto m \cdot x \in \mathcal{X}
$$

extends to a completely contractive $w^{*}$-continuous map from $\mathcal{M} \otimes_{\sigma h} \mathcal{X}$ into $\mathcal{X}$.

As $\mathcal{X}$ is the dual space, there is an operator space $\mathcal{Y}$ such that $\mathcal{X}=$ $\mathcal{Y}^{*}$. As $\mathcal{X}$ is a normal operator $\mathcal{M}$-module, $\mathcal{Y}^{* *}=\mathcal{X}^{*}$ can be turned canonically into a Banach $\mathcal{M}$-bimodule and then it is easy to check that $\mathcal{Y}$ is a Banach sub- $\mathcal{M}$-module of $\mathcal{Y}^{* *}$. Hence, normal operator modules are the operator space analog of the normal dual Banach modules (see $[23])$.

Lemma 2.2. Let $\mathcal{M}$ be a unital dual operator algebra. Then, $\mathcal{M} \otimes_{\sigma h} \mathcal{M}$ is a normal operator $\mathcal{M}$-bimodule.

Proof. We give here a quick proof, see Lemma 2.5 in [1]. For simplicity, we just prove this for the left action of $\mathcal{M}$. It is sufficient to prove that $\alpha: \mathcal{M} \otimes\left(\mathcal{M} \otimes_{\sigma h} \mathcal{M}\right) \rightarrow \mathcal{M} \otimes_{\sigma h} \mathcal{M}$ defined by $\alpha(m \otimes$ $v)=m \cdot v$ extends to a completely contractive $w^{*}$-continuous map on $\mathcal{M} \otimes_{\sigma h}\left(\mathcal{M} \otimes_{\sigma h} \mathcal{M}\right)$. This dual space (by associativity of $\otimes_{\sigma h}$ ) can be identified with $\left(\mathcal{M} \otimes_{\sigma h} \mathcal{M}\right) \otimes_{\sigma h} \mathcal{M}$ via a $w^{*}$-continuous complete isometry. Recall that the action of $\mathcal{M}$ on $\mathcal{M} \otimes_{\sigma h} \mathcal{M}$ is determined by

$$
m \cdot(x \otimes y)=(m x) \otimes y,
$$

hence

$$
\alpha_{\mid \mathcal{M} \otimes \mathcal{M} \otimes \mathcal{M}}=\left(\mathrm{m}_{\mathcal{M}} \otimes_{\sigma h} \mathrm{id}_{\mathcal{M}}\right)_{\mid \mathcal{M} \otimes \mathcal{M} \otimes \mathcal{M}}
$$

So $\alpha$ has a completely contractive $w^{*}$-continuous extension to $\mathcal{M} \otimes_{\sigma h}$ $\mathcal{M} \otimes_{\sigma h} \mathcal{M}$.

This previous Lemma can not be stated with the normal (or binormal) projective tensor product (see [8] p. 139). The previous proof actually relies on the associativity of the normal Haagerup tensor product.

The 'only if' part of the next proposition is still true for dual Banach algebras, whereas the 'if' part is not (see [21]). 
Following notation of [23], when $\mathcal{X}$ is a normal operator $\mathcal{M}$-bimodule, we denote $H_{w c b}^{1}(\mathcal{M}, \mathcal{X})$ the first Hochschild cohomology group relatively to the normal completely bounded multi-linear maps.

Proposition 2.3. Let $\mathcal{M}$ be a unital dual operator algebra. Then, $\mathcal{M}$ admits a $\sigma$-diagonal if and only if $H_{w c b}^{1}(\mathcal{M}, \mathcal{X})=0$, for every normal operator $\mathcal{M}$-bimodule $\mathcal{X}$.

Proof. The proof follows B.E. Johnson's proof on characterization of amenable Banach algebra in terms of virtual diagonal, so we sketch it (for more details, see e.g. Lemma 7.4.4 in [2]). Suppose $u$ is a $\sigma h$-diagonal for $\mathcal{M}$ and let $D: \mathcal{M} \rightarrow \mathcal{X}$ be a completely bounded $w^{*}$ continuous derivation into a normal operator $\mathcal{M}$-bimodule $\mathcal{X}$. As $\otimes_{\sigma h}$ we can consider $D \otimes_{\sigma h} \mathrm{id}_{\mathcal{M}}$ and then define $\varphi=D \otimes_{\sigma h} \operatorname{id}_{\mathcal{M}}(u)$. It is not difficult to check that $D$ is the inner derivation implemented by $\varphi$. For the converse, note that $\operatorname{Ker}_{\mathcal{M}}$ is a normal operator sub- $\mathcal{M}$-bimodule of $\mathcal{M} \otimes_{\sigma h} \mathcal{M}$ (see Lemma 2.2 above), so we can follow the computation of Lemma 7.4 .4 in [2].

We recall from [19] that two representations of a dual operator algebra, admitting a $\sigma h$-diagonal, which are close enough are necessarily similar.

If $S \in \mathbb{B}(H)$ is an invertible operator, we denote $\operatorname{Ad}_{S}$ the similarity implemented by $S$.

Proposition 2.4. Let $\mathcal{M}$ be a unital dual operator algebra. We suppose that $\mathcal{M}$ has a $\sigma$ h-diagonal $u \in \mathcal{M} \otimes_{\sigma h} \mathcal{M}$. Let $\pi_{1}, \pi_{2}$ be two unital $w^{*}$-continuous completely bounded homomorphism into a same dual operator algebra $\mathcal{N}$.

If $\left\|\pi_{1}-\pi_{2}\right\|_{c b}<\|u\|^{-1} \max \left\{\left\|\pi_{1}\right\|_{c b}^{-1},\left\|\pi_{2}\right\|_{c b}^{-1}\right\}$, then there exists an invertible operator $S$ in the $w^{*}$-closed algebra generated by $\pi_{1}(\mathcal{M}) \cup \pi_{2}(\mathcal{M})$ such that $\pi_{1}=\operatorname{Ad}_{S} \circ \pi_{2}$.

Proof. See Proposition 4.1 [19], noticing that the proof works if one replaces $\mathbb{B}(K)$ by any arbitrary unital dual operator algebras.

Remark 2.5. Note that the previous Proposition works in the category of unital amenable Banach algebra with unital bounded homomorphisms (or unital dual Banach algebras with $w^{*}$-continuous unital bounded homomorphisms), see [20].

Now we are going to see that the converse of the previous proposition is true.

Let $\mathcal{M}, \mathcal{N}$ be two unital dual operator algebras, we denote $\operatorname{Hom}_{c b w^{*}}(\mathcal{M}, \mathcal{N})$ the set of all unital $w^{*}$-continuous completely bounded homomorphisms 
equipped with the metric induced by the cb-norm i.e. the distance between $\pi_{1}, \pi_{2} \in \operatorname{Hom}_{c b w^{*}}(\mathcal{M}, \mathcal{N})$ is defined by $\left\|\pi_{1}-\pi_{2}\right\|_{c b}$. For $\pi \in$ $\operatorname{Hom}_{c b w^{*}}(\mathcal{M}, \mathcal{N})$, we also denote $[\pi]$ the similarity orbit of $\pi$, that is

$$
[\pi]=\left\{\operatorname{Ad}_{S} \circ \pi, S \in \mathcal{N}^{-1}\right\} \subset \operatorname{Hom}_{c b w^{*}}(\mathcal{M}, \mathcal{N}),
$$

where $\mathcal{N}^{-1} \subset \mathcal{N}$ denotes the group of invertible operators in $\mathcal{N}$.

Proposition 2.6. Let $\mathcal{M}$ be a unital dual operator algebra. Suppose that for any unital dual operator algebra $\mathcal{N}$, for any $\pi \in \operatorname{Hom}_{\text {cbw* }}(\mathcal{M}, \mathcal{N})$, $[\pi]$ is open, then $\mathcal{M}$ admits a oh-diagonal.

Proof. Let $\mathcal{X}$ be a normal operator $\mathcal{M}$-bimodule and $D: \mathcal{M} \rightarrow \mathcal{X}$ be a $w^{*}$-continuous completely bounded derivation. From Proposition 2.3, we must show that $D$ is inner. Let $\mathcal{N}$ be the space of all elements of the form

$$
\left[\begin{array}{cc}
m & x \\
0 & m
\end{array}\right],
$$

where $m \in \mathcal{M}$ and $x \in \mathcal{X}$. Actually, using the module actions, we can equipped $\mathcal{N}$ with a multiplication. Then $\mathcal{N}$ becomes a unital dual operator algebra (arguing as in Section 4 of [1] ). Now, denote $\pi=\operatorname{id}_{\mathcal{M}} \oplus \operatorname{id}_{\mathcal{M}}: \mathcal{M} \rightarrow \mathcal{N}$, which is obviously a unital $w^{*}$-continuous completely bounded homomorphism. Hence there is an $\varepsilon>0$ such that the open ball of radius $\varepsilon$ centered in $\pi$ is contained in $[\pi]$. Put $r=\frac{\varepsilon}{2\|D\|_{c b}}$ and define $\varrho: \mathcal{M} \rightarrow \mathcal{N}$ by

$$
\varrho(m)=\left[\begin{array}{cc}
m & r D(m) \\
0 & m
\end{array}\right]
$$

So $\varrho$ is a unital $w^{*}$-continuous completely bounded homomorphism from $\mathcal{M}$ into $\mathcal{N}$ and

$$
\|\pi-\varrho\|_{c b}<\varepsilon
$$

Consequently, there exists an invertible

$$
S=\left[\begin{array}{cc}
m & x \\
0 & m
\end{array}\right] \in \mathcal{N}
$$

such that $\pi=\operatorname{Ad}_{S} \circ \varrho$. Easy computation show that $D$ is in fact the inner derivation implemented by $\frac{1}{r} x m^{-1}$.

To summarize:

Corollary 2.7. Let $\mathcal{M}$ be a unital dual operator algebra. The following are equivalent:

(i) $\mathcal{M}$ has a $\sigma h$-diagonal $u \in \mathcal{M} \otimes_{\sigma h} \mathcal{M}$,

(ii) for every normal operator $\mathcal{M}$-bimodule $\mathcal{X}, H_{w c b}^{1}(\mathcal{M}, \mathcal{X})=0$, 
(iii) for any unital dual operator algebra $\mathcal{N}$, for any $\pi \in \operatorname{Hom}_{\text {cbw* }}(\mathcal{M}, \mathcal{N})$, the similarity orbit $[\pi]$ is clopen.

It is clear that the class of $\sigma h$-amenable operator algebras are stable under unital $w^{*}$-continuous cb-homomorphism with $w^{*}$-closed range. Also $w^{*}$-closed ideals of a $\sigma h$-amenable operator algebra are $\sigma h$-amenable as well. More precisely:

Proposition 2.8. Let $\mathcal{M}$ be a unital dual operator algebra and $\mathcal{I}$ be a $w^{*}$-closed ideal of $\mathcal{M}$.

(1) If $\mathcal{M}$ is $\sigma$-amenable (with diagonal $u$ ), then there is a unique central idempotent $p$ such that $\mathcal{I}=p \mathcal{M}$. Moreover, $\|p\| \leq\|u\|$. Consequently, $\mathcal{I}$ and $\mathcal{M} / \mathcal{I}$ are $\sigma$ h-amenable.

(2) Conversely, if $\mathcal{I}$ and $\mathcal{M} / \mathcal{I}$ are $\sigma$ h-amenable then $\mathcal{M}$ is $\sigma h$ amenable.

Proof. For (1), by Proposition 7.4.12 in [2], $\mathcal{M}$ has the $w^{*}$-module complementation property, so we can adapt the proof of Theorem 3.2.2 in [10]. For (2), see Theorem 2.3.10 in [20].

\section{Perturbation of $\sigma h$-amenable dual operator algebras}

3.1. Algebraic perturbations. This subsection is inspired by Theorem 6.2 in [12], where B.E. Johnson proves the stability of amenability for neighboring multiplications (that we call here algebraic perturbations). One can prove the analog for a $\sigma h$-amenable operator algebra $\mathcal{M}$ by first noticing that every Hochschild cohomology groups $H_{w c b}^{k}(\mathcal{M}, \mathcal{M})$ (associated to the normal completely bounded cohomology) actually vanish, and then applying Theorem 1 of [18].

In the following, a bilinear $\mathrm{m}: \mathcal{M} \times \mathcal{M} \rightarrow \mathcal{M}$ is called a multiplication on $\mathcal{M}$ if it is associative, extends to the normal Haagerup tensor product $\mathcal{M} \otimes_{\sigma h} \mathcal{M}$ and unital (i.e. $\mathrm{m}(1, x)=\mathrm{m}(x, 1)=x$ ). We know that given a multiplication $\mathrm{m}$ (from [2] Theorems 5.2.1 and 5.2.16), that $\mathcal{M}$ equipped with $\mathrm{m}$ is cb-isomorphic via a unital $w^{*}$-homeomorphism to an actual dual operator algebra. Therefore, it makes sense to talk about the $\sigma h$-amenability of $(\mathcal{M}, \mathrm{m})$. We denote by $\mathrm{m}_{\mathcal{M}}$ the original multiplication on $\mathcal{M}$.

Lemma 3.1. Let $\mathcal{M}$ be a unital $\sigma$ h-amenable dual operator algebra. Then, $H_{w c b}^{n}(\mathcal{M}, \mathcal{M})=0$, for any $n$.

Proof. Firstly, using the $\sigma h$-diagonal to average, one can prove the analog of Theorem 1.7.4 in [23]. Denote $C B_{\sigma}(\mathcal{M}, \mathcal{M})$ the set of all $w^{*}$-continuous completely bounded linear maps from $\mathcal{M}$ into $\mathcal{M}$ and 
denote $C B_{\sigma}(\mathcal{M}, \mathcal{M})_{\mathcal{M}}$ the subspace of $C B_{\sigma}(\mathcal{M}, \mathcal{M})$ consisting of the right $\mathcal{M}$-module maps. Let $L \in C B_{\sigma}(\mathcal{M}, \mathcal{M})$, consider

$$
\Pi_{L}: \mathcal{M} \otimes_{\sigma h} \mathcal{M} \otimes_{\sigma h} \mathcal{M} \rightarrow \mathcal{N}
$$

defined

$$
\Pi_{L}(x \otimes y \otimes z)=L(x y) z .
$$

Now, for $m \in \mathcal{M}$, we define $\Pi(L)(m)=\Pi_{L}(m \otimes u)$, where $u$ is a $\sigma h$-diagonal. Then one can check that $\Pi$ is a completely bounded projection from $C B_{\sigma}(\mathcal{M}, \mathcal{M})$ onto $C B_{\sigma}(\mathcal{M}, \mathcal{M})_{\mathcal{M}}$ with $\|\Pi\|_{c b} \leq\|u\|$. Secondly, as $u \in \mathcal{M} \otimes_{\sigma h} \mathcal{M}$, there is a net $\left(u_{t}\right)_{t}$ in $\mathcal{M} \otimes \mathcal{M}$ converging to $u$ in the $w^{*}$-topology of $\mathcal{M} \otimes_{\sigma h} \mathcal{M}$. For any $t$, there are finite families $\left(a_{k}^{t}\right)_{k},\left(b_{k}^{t}\right)_{k}$ of elements in $\mathcal{M}$ such that $u=w^{*}-\lim _{t} \sum_{k} a_{k}^{t} \otimes b_{k}^{t}$. Then one can follow the computation of Theorem 7.3.1 in [23] in replacing $\sum_{j} m_{j}^{\alpha *} \otimes m_{j}^{\alpha}$ by $u=w^{*}-\lim _{t} \sum_{k} a_{k}^{t} \otimes b_{k}^{t}$.

Remark 3.2. Note that $w^{*}$-continuity of $\Pi(L)$ actually comes from the associativity of the normal Haagerup tensor product. There might be another way to prove this Lemma using a reduction of dimension argument, but computations might be longer.

Note that it is not too difficult to state the analog of Theorem 3 of [18] (or Theorem 2.1 of [12], see also Theorem 7.4.1 in [23]) for a dual operator algebra $\mathcal{M}$. One has to replace hypothesis on the continuous cohomology by the same hypothesis on the normal completely bounded cohomology i.e.

$$
H_{w c b}^{2}(\mathcal{M}, \mathcal{M})=H_{w c b}^{3}(\mathcal{M}, \mathcal{M})=0
$$

and replace the norm of both multiplications by their cb-norm i.e.

$$
\left\|\mathrm{m}-\mathrm{m}_{\mathcal{M}}\right\|_{c b} \text {. }
$$

The conclusion is the existence of constants $\delta, C>0$ and existence of a $w^{*}$-continuous completely bounded linear isomorphism $\Phi$ of $\mathcal{M}$ satisfying

$$
\left\|\Phi-\mathrm{id}_{\mathcal{M}}\right\|_{c b} \leq C\left\|\mathrm{~m}-\mathrm{m}_{\mathcal{M}}\right\|_{c b} \text { and } \Phi(\mathrm{m}(x, y))=\Phi(x) \Phi(y),
$$

providing that $\left\|\mathrm{m}-\mathrm{m}_{\mathcal{M}}\right\|_{c b} \leq \delta$.

For the proof of this fact, one just has to apply the implicit function Theorem 1 in [18] (see also Theorem 7.3.1 in [23]) to the right spaces of $w^{*}$-continuous completely bounded multilinear maps. As a corollary, if $\mathcal{M}$ is $\sigma h$-amenable, then $(\mathcal{M}, \mathrm{m})$ is also $\sigma h$-amenable (because $\Phi$ is a unital $w^{*}$-continuous cb-isomorphism between these algebras). The flaw in this -too general- approach is that the constant $\delta$ is implicit. Here we give another argument using [19] which enables us to explicit a bound. 
Recall that $T^{\vee}$ denotes the bilinear map from $\mathcal{M} \times \mathcal{M}$ into $\mathcal{N}$ defined by $T^{\vee}(x, y)=T(x y)-T(x) T(y)$.

Theorem 3.3. Let $\mathcal{M}, \mathcal{N}$ be two unital dual operator algebras. We suppose that $\mathcal{M}$ has a normal virtual h-diagonal $u \in \mathcal{M} \otimes_{\sigma h} \mathcal{M}$. Then, for any $\varepsilon \in] 0,1[$, for any $\mu>0$, there exists $\delta>0$ such that: for every unital $w^{*}$-continuous linear map $L: \mathcal{M} \rightarrow \mathcal{N}$ satisfying $\|L\|_{c b} \leq \mu$ and $\left\|L^{\vee}\right\|_{c b} \leq \delta$, there is a unital $w^{*}$-continuous completely bounded algebra homomorphism $\pi: \mathcal{M} \rightarrow \mathcal{N}$ such that $\|L-\pi\|_{c b} \leq \varepsilon$.

Proof. See [19], we just recall that one can choose $\delta=\frac{\varepsilon}{4\|u\|+8 \mu^{2}\|u\|^{2}}$.

Let us recall more precisely Theorems 5.2.1 and 5.2.16 in [2]. Given a completely bounded multiplication $\mathrm{m}$ on $\mathcal{M}$, there exists a dual operator algebra $\mathcal{N}$ and a completely bounded unital $w^{*}$-continous algebra homomorphism $\rho: \mathcal{M} \rightarrow \mathcal{N}$ with $\|\rho\|_{c b} \leq 2\|\mathrm{~m}\|_{c b}$ and $\left\|\rho^{-1}\right\|_{c b} \leq$ $\|\mathrm{m}\|_{c b}^{-1}$.

Proposition 3.4. Let $\mathcal{M}$ be a unital $\sigma$ h-amenable dual operator algebra (with $\sigma$ h-diagonal $u$ ). Let $\mathrm{m}$ be another multiplication on $\mathcal{M}$. If $\left\|\mathrm{m}_{\mathcal{M}}-\mathrm{m}\right\|_{c b} \leq \frac{23}{192\|u\|+1536\|u\|^{2}}$, then $(\mathcal{M}, \mathrm{m})$ is oh-amenable.

Proof. Define $L=\rho \circ \operatorname{id}_{\mathcal{M}}: \mathcal{M} \rightarrow \mathcal{N}$ (with notation of the preceding discussion). Note that

$$
\left\|L^{\vee}\right\|_{c b} \leq\|\rho\|_{c b}\left\|\mathrm{~m}_{\mathcal{M}}-\mathrm{m}\right\|_{c b} \leq 2\|\mathrm{~m}\|_{c b}\left\|\mathrm{~m}_{\mathcal{M}}-\mathrm{m}\right\|_{c b} .
$$

We can to apply the previous theorem to $L: \mathcal{M} \rightarrow \mathcal{N}$. Let $\delta=$ $2\|\mathrm{~m}\|_{c b}\left\|\mathrm{~m}_{\mathcal{M}}-\mathrm{m}\right\|_{c b}, \mu=2\|\mathrm{~m}\|_{c b}$ and recall from the proof of Theorem 3.2 in [19] that $\varepsilon=\delta\left(4\|u\|+8 \mu^{2}\|u\|^{2}\right)$. Here we need to require $\varepsilon<1$, so that the resulting algebra homomorphism $\pi$ is bijective, hence $\sigma$-amenability of $\left(\mathcal{M}, \mathrm{m}_{\mathcal{M}}\right)$ transfers to $\mathcal{N}$ and consequently to $(\mathcal{M}, \mathrm{m})$. In order to apply the previous theorem, we need the following condition to be satisfied:

$$
2\|\mathrm{~m}\|_{c b}\left\|\mathrm{~m}_{\mathcal{M}}-\mathrm{m}\right\|_{c b} \leq \frac{1}{4\|u\|+32\|\mathrm{~m}\|_{c b}^{2}\|u\|^{2}} .
$$

But using the obvious fact $\|\mathrm{m}\|_{c b} \leq 1+\left\|\mathrm{m}_{\mathcal{M}}-\mathrm{m}\right\|_{c b}$, we obtain that the previous condition is satisfied if

$$
\left\|\mathrm{m}_{\mathcal{M}}-\mathrm{m}\right\|_{c b}\left(1+\left\|\mathrm{m}_{\mathcal{M}}-\mathrm{m}\right\|_{c b}\right)^{3} \leq \frac{1}{8\|u\|+64\|u\|^{2}} .
$$

As $\|u\| \geq 1$, this last condition would also imply that $\left\|\mathrm{m}_{\mathcal{M}}-\mathrm{m}\right\|_{c b} \leq$ $\frac{1}{72}$. Finally, if

$$
\left\|\mathrm{m}_{\mathcal{M}}-\mathrm{m}\right\|_{c b} \leq \frac{23}{192\|u\|+1536\|u\|^{2}}
$$


we can apply the previous theorem and conclude.

Lemma 3.5. For any $\eta>0$, there exists $\rho \in(0,1)$ such that for any unital operator algebras $\mathcal{A}, \mathcal{B}$, for any unital cb-isomorphism $T: \mathcal{A} \rightarrow$ $\mathcal{B},\|T\|_{c b} \leq 1+\rho$ and $\left\|T^{-1}\right\|_{c b} \leq 1+\rho$ imply $\left\|T^{\vee}\right\|_{c b}<\eta$.

Proof. Suppose the assertion is false, then there exists $\eta_{o}>0$ such that for every positive integer $n \in \mathbb{N}^{*}$, there is a unital cb-isomorphism $T_{n}$ : $\mathcal{A}_{n} \rightarrow \mathcal{B}_{n}$ between some unital operator algebras satisfying $\left\|T_{n}\right\|_{c b} \leq$ $1+1 / n,\left\|T_{n}^{-1}\right\|_{c b} \leq 1+1 / n$ and $\left\|T_{n}^{\vee}\right\|_{c b} \geq \eta_{o}$. Let $\mathcal{U}$ be a nontrivial ultrafilter on $\mathbb{N}$, let us denote $\mathbb{K}^{1}$ the unitization of the $C^{*}$-algebra of all compact operators on $l_{2}$. Denote also $\mathcal{A}_{\mathcal{U}}\left(\operatorname{resp} . \mathcal{B}_{\mathcal{U}}\right)$ the ultraproduct $\Pi_{n} \mathbb{K}^{1} \otimes_{\min } \mathcal{A}_{n} / \mathcal{U}$ (resp. $\left.\Pi_{n} \mathbb{K}^{1} \otimes_{\min } \mathcal{B}_{n} / \mathcal{U}\right)$, this is a unital operator algebra (see [2] Chapter 2). Now consider $T_{\mathcal{U}}: \mathcal{A}_{\mathcal{U}} \rightarrow \mathcal{B}_{\mathcal{U}}$ the ultraproduct map obtained from the $\mathrm{id}_{\mathbb{K}^{1}} \otimes T_{n}$ 's. Hence $T_{\mathcal{U}}$ is a unital linear complete isometry between operator algebras, so $T_{\mathcal{U}}$ is multiplicative (see [2] Theorem 4.5.13). This contradicts the hypothesis for all $n,\left\|T_{n}^{\vee}\right\|_{c b} \geq \eta_{o}$. Because $\left\|T_{n}^{\vee}\right\|_{c b}=\left\|\left(\mathrm{id}_{\mathbb{K}^{1}} \otimes T_{n}\right)^{\vee}\right\|$, so the condition $\left\|T_{n}^{\vee}\right\|_{c b} \geq \eta_{o}$ means that there are $x_{n}, y_{n}$ in the closed unit ball of $\mathbb{K}^{1} \otimes_{\min } \mathcal{A}_{n}$ such that

$$
\left\|\left(\mathrm{id}_{\mathbb{K}^{1}} \otimes T_{n}\right)\left(x_{n} y_{n}\right)-\left(\mathrm{id}_{\mathbb{K}^{1}} \otimes T_{n}\right)\left(x_{n}\right)\left(\mathrm{id}_{\mathbb{K}^{1}} \otimes T_{n}\right)\left(y_{n}\right)\right\| \geq \eta_{o},
$$

which implies that

$$
\left\|T_{\mathcal{U}}(\dot{x} \dot{y})-T_{\mathcal{U}}(\dot{x}) T_{\mathcal{U}}(\dot{y})\right\| \geq \eta_{o}
$$

where $\dot{x}$ (resp. $\dot{y}$ ) denotes the equivalence class of $\left(x_{n}\right)_{n}\left(\operatorname{resp} .\left(y_{n}\right)_{n}\right)$ in $\mathcal{A}_{\mathcal{U}}$. This last inequality contradicts the multiplicativity of $T_{\mathcal{U}}$ (this ultraproduct argument is inspired from [11]).

Theorem 3.6. There exists a function $f:(1,+\infty) \rightarrow(0,1)$ such that for every $\sigma$ h-amenable unital dual operator algebra $\mathcal{M}$ (with $\sigma$ hdiagonal $u$ ), for any unital dual operator algebra $\mathcal{N}$, for any unital $w^{*}$-continuous linear cb-isomorphism $T: \mathcal{M} \rightarrow \mathcal{N}$, the inequalities $\|T\|_{c b} \leq 1+f(\|u\|)$ and $\left\|T^{-1}\right\|_{c b} \leq 1+f(\|u\|)$ imply that $\mathcal{M}$ and $\mathcal{N}$ are cb-isomorphic via a unital $w^{*}$-continuous algebra homomorphism (in particular, $\mathcal{N}$ is $\sigma$ h-amenable).

Proof. Let $\mathcal{M}$ be a unital dual operator algebra admitting a $\sigma h$ diagonal $u$. Applying Theorem 3.3 with $\mu=2, \varepsilon=1 / 2$ gives a $\delta$ (as function of $\|u\|$ ). Now we can apply previous Lemma with $\eta=\delta$ to obtain $\rho$ and so we can define $f(\|u\|)=\rho$. Because if $T: \mathcal{M} \rightarrow \mathcal{N}$ is a unital $w^{*}$-continuous linear cb-isomorphism satisfying $\|T\|_{c b} \leq 1+f(\|u\|)$ and $\left\|T^{-1}\right\|_{c b} \leq 1+f(\|u\|)$, hence $\left\|T^{\vee}\right\|_{c b} \leq \delta$. Therefore by Theorem 
3.3, there exists a unital $w^{*}$-continuous completely bounded algebra homomorphism $\pi: \mathcal{M} \rightarrow \mathcal{N}$ such that $\|T-\pi\|_{c b} \leq 1 / 2$. Hence $\left\|T^{-1} \pi-\operatorname{id}_{\mathcal{M}}\right\|_{c b}<1, \pi$ is bijective and $\left\|\pi^{-1}\right\|_{c b} \leq\left\|T^{-1}\right\|_{c b}\left\|\pi^{-1} T\right\|_{c b} \leq$ $\frac{\left\|T^{-1}\right\|_{c b}}{1-\left\|T^{-1} \pi-\operatorname{id}\right\|_{\mathcal{M}} \|_{c b}}$.

Remark 3.7. It would be ideal to prove a non-unital version of the previous theorem involving the Banach-Mazur cb-distance.

3.2. Perturbation results for the Kadison-Kastler metric. Let $H$ be an Hilbert space, and $\mathbb{B}(H)$ the von Neumann algebra of all bounded operators on $H$. Let $\mathcal{E}, \mathcal{F}$ be two subsets of $\mathbb{B}(H)$. Let $\gamma>0$, we write $\mathcal{E} \subseteq^{\gamma} \mathcal{F}$ if for any $x$ in the unit ball of $\mathcal{E}$, there exists $y$ in $\mathcal{F}$ such that

$$
\|x-y\| \leq \gamma .
$$

We also write $\mathcal{E} \subset^{\gamma} \mathcal{F}$ if there exists $\gamma^{\prime}<\gamma$ such that $\mathcal{E} \subseteq \gamma^{\prime} \mathcal{F}$. We will need the notion of near cb-inclusion. As usual in operator space theory, $\mathbb{M}_{n}(\mathcal{E})$, the set of $n \times n$ matrices with coefficients in $\mathcal{E}$ is normed by the identification $\mathbb{M}_{n}(\mathcal{E}) \subset \mathbb{M}_{n}(\mathbb{B}(H))=\mathbb{B}\left(\ell_{n}^{2} \otimes H\right)$. Finally, we write $\mathcal{E} \subseteq_{c b}^{\gamma} \mathcal{F}$ if, for any $n, \mathbb{M}_{n}(\mathcal{E}) \subseteq^{\gamma} \mathbb{M}_{n}(\mathcal{F})$.

In Theorem 5.2 in [19], $\mathcal{N}$ is injective i.e. $\mathcal{N}$ is the range of a completely contractive projection. The purpose of this subsection would be to parametrize Theorem 5.2 in [19] in function of the cb-norm of the projection.

Lemma 3.8. Let $\mathcal{M}, \mathcal{N} \subset \mathbb{B}(H)$ be two unital $w^{*}$-closed operator algebras. We suppose that $\mathcal{N}$ is the range of completely bounded $w^{*}$ continuous projection $P$. Denote $T=P_{\mid \mathcal{M}}: \mathcal{M} \rightarrow \mathcal{N}$.

If $\mathcal{M} \subseteq_{c b}^{\gamma} \mathcal{N}$, with

$$
\gamma<\left(1+\|P\|_{c b}\right)^{-1},
$$

then $T$ is injective and satisfies:

(1) $\left\|T-\operatorname{id}_{\mathcal{M}}\right\|_{c b} \leq\left(1+\|P\|_{c b}\right) \gamma$

(2) $\left\|T^{\vee}\right\|_{c b} \leq\left(1+\|P\|_{c b}\right)\left(2+\|P\|_{c b}\right) \gamma$, where $T^{\vee}$ is the bilinear map from $\mathcal{M} \times \mathcal{M}$ into $\mathcal{N}$ defined by $T^{\vee}(x, y)=T(x y)-T(x) T(y)$.

Moreover, if $\mathcal{N} \subset \frac{1}{\|P\|} \mathcal{M}$, then $T$ is bijective and

$$
\left\|T^{-1}\right\|_{c b} \leq \frac{1}{1-\left(1+\|P\|_{c b}\right) \gamma} .
$$


Proof. Let $x$ in the unit ball of $\mathbb{M}_{n}(\mathcal{M})$, then there is $y$ in $\mathbb{M}_{n}(\mathcal{N})$ such that $\|x-y\| \leq \gamma$.

$$
\left\|T_{n}(x)-x\right\|=\left\|T_{n}(x-y)-(x-y)\right\|_{c b} \leq\left(1+\|P\|_{c b}\right) \gamma .
$$

which proves (1).

For (2), let $x, y$ in the unit ball of $\mathbb{M}_{n}(\mathcal{M})$

$$
\begin{aligned}
\left(T^{\vee}\right)_{n}(x, y) & =T_{n}(x y)-T_{n}(x) T_{n}(y) \\
& =T_{n}(x y)-x y \\
& +x y-x T_{n}(y) \\
& +x T_{n}(y)-T_{n}(x) T_{n}(y)
\end{aligned}
$$

Consequently, using (1), we obtain

$$
\begin{aligned}
\left\|T^{\vee}\right\|_{c b} & \leq\left\|T-\operatorname{id}_{\mathcal{M}}\right\|_{c b}+\left\|T-\operatorname{id}_{\mathcal{M}}\right\|_{c b}+\|T\|_{c b}\left\|T-\operatorname{id}_{\mathcal{M}}\right\|_{c b} \\
& \leq\left(1+\|P\|_{c b}\right)\left(2+\|P\|_{c b}\right) \gamma,
\end{aligned}
$$

which gives (2). Now let's prove that $T$ is injective. Let $x$ in $\mathbb{M}_{n}(\mathcal{M})$. From (1) and $\gamma \leq\left(1+\|P\|_{c b}\right)^{-1}$, we have

$$
\begin{aligned}
\left\|T_{n}(x)\right\| & \geq\left|\left\|T_{n}(x)-x\right\|-\|x\|\right| \\
& \geq\left(1-\left(1+\|P\|_{c b}\right) \gamma\right)\|x\|
\end{aligned}
$$

so $T$ is injective (and has closed range).

Now assume $\mathcal{N} \subset \frac{1}{\|P\|} \mathcal{M}$. To conclude we just need to prove that $T$ is surjective, because the inequality

$$
\left\|T^{-1}\right\|_{c b} \leq \frac{1}{1-\left(1+\|P\|_{c b}\right) \gamma}
$$

will follow from the inequality we have just proved. Let $\gamma^{\prime}<\|P\|^{-1}$ such that $\mathcal{N} \subseteq{ }^{\gamma^{\prime}} \mathcal{M}$. Let $y$ in the unit ball of $\mathcal{N}$, then there exists $x$ in $\mathcal{M}$ such that $\|y-x\| \leq \gamma^{\prime}$. Hence

$$
\|y-T(x)\|=\|T(y-x)\| \leq \gamma^{\prime}\|T\| \leq \gamma^{\prime}\|P\| .
$$

Denote $t_{1}=T(x)$, which is in the range of $T$. Applying the same procedure to $\frac{1}{\gamma^{\prime}\|P\|}\left(y-t_{1}\right)$ (which is the unit ball of $\mathcal{N}$ ), we obtain $t_{2}$ in the range of $T$ such that

$$
\left\|y-t_{1}-t_{2}\right\| \leq\left(\gamma^{\prime}\|P\|\right)^{2}
$$

Inductively, for any $j$, we can find $t_{1}, \ldots, t_{j}$ in the range of $T$ such that

$$
\left\|y-\left(t_{1}+t_{2}+\cdots+t_{j}\right)\right\| \leq\left(\gamma^{\prime}\|P\|\right)^{j} .
$$


As $\gamma^{\prime}<\|P\|^{-1}$, we conclude that $y$ belongs to the closure of the range of $T$. But we have seen that $T$ has closed range, so finally $T$ is surjective.

For the following Theorem, we denote by $F$ the polynomial function

$$
F(X, Y)=(1+X)\left(Y+4(X+2) Y^{2}+8(X+2) X^{2} Y^{3}\right)
$$

The proof is similar to the proof of Theorem 5.5 in [19].

Theorem 3.9. Let $\mathcal{M}, \mathcal{N} \subset \mathbb{B}(H)$ be two unital $w^{*}$-closed operator algebras. We suppose that $\mathcal{M}$ has a $\sigma$ h-diagonal $u \in \mathcal{M} \otimes_{\sigma h} \mathcal{M}$. We suppose that $\mathcal{N}$ is the range of a completely bounded $w^{*}$-continuous projection $P$.

If $\mathcal{N} \subset \frac{1}{\|P\|} \mathcal{M}$ and $\mathcal{M} \subseteq_{c b}^{\gamma} \mathcal{N}$, with $\gamma<1 / F\left(\|P\|_{c b},\|u\|\right)$, then there exists an invertible operator $S$ in the $w^{*}$-closed algebra generated by $\mathcal{M} \cup \mathcal{N}$ such that $S \mathcal{M} S^{-1}=\mathcal{N}$. Moreover,

$$
\left\|S-I_{H}\right\| \leq \gamma F\left(\|P\|_{c b},\|u\|\right) .
$$

Proof. As $\|u\| \geq 1$, we have $\gamma<\left(1+\|P\|_{c b}\right)^{-1}$. Hence, by Lemma 3.8, there is a unital completely bounded $w^{*}$-continuous linear bijection $T$ from $\mathcal{M}$ onto $\mathcal{N}$ such that

$$
\left\|T^{\vee}\right\|_{c b} \leq\left(1+\|P\|_{c b}\right)\left(2+\|P\|_{c b}\right) \gamma \text { and }\|T\|_{c b} \leq\|P\|_{c b} .
$$

Let

$$
\varepsilon=\left(1+\|P\|_{c b}\right)\left(\left(4\|P\|_{c b}+8\right)\|u\|+\left(8\|P\|_{c b}+16\right)\|P\|_{c b}^{2}\|u\|^{2}\right) \gamma .
$$

Note that from the condition on $\gamma$, we see that

$$
\varepsilon+\left(1+\|P\|_{c b}\right) \gamma<\|u\|^{-1} \leq 1
$$

We also write $\mu=\|P\|_{c b}$ and $\delta=\left(4\|u\|+8 \mu^{2}\|u\|^{2}\right)^{-1} \varepsilon$. So, by definition of $\varepsilon$,

$$
\delta=\left(1+\|P\|_{c b}\right)\left(2+\|P\|_{c b}\right) \gamma
$$

From Equations (3.2) and (3.3) above, we can apply Theorem 3.3 to the linear map $T$. Thus there exists a unital $w^{*}$-continuous completely bounded homomorphism $\pi: \mathcal{M} \rightarrow \mathcal{N}$ such that $\|T-\pi\|_{c b} \leq \varepsilon$. Consequently

$$
\begin{aligned}
\left\|\pi-\mathrm{id}_{\mathcal{M}}\right\|_{c b} & \leq\|\pi-T\|_{c b}+\left\|T-\mathrm{id}_{\mathcal{M}}\right\|_{c b} \\
& \leq \varepsilon+\left(1+\|P\|_{c b}\right) \gamma
\end{aligned}
$$

From equation (3.3), we have for any $x \in \mathcal{M}$,

$$
\|\pi(x)\| \geq\left(1-\left(\varepsilon+\left(1+\|P\|_{c b}\right) \gamma\right)\|x\|,\right.
$$


so $\pi$ is injective and has closed range. For the surjectivity, let $y$ in the unit ball of $\mathcal{N}$. Put $x=\pi\left(T^{-1}(y)\right.$ ). Further, we have (from Lemma $(3.8))$

$$
\|y-x\| \leq\|T-\pi\|\left\|T^{-1}(y)\right\| \leq \frac{\varepsilon}{1-\left(1+\|P\|_{c b}\right) \gamma} .
$$

Inductively, as in the proof of Lemma 3.8, we have that for any $j$, there are $x_{j}$ in the range of $\pi$ such that $\left\|y-x_{j}\right\| \leq\left(\varepsilon\left(1-\left(1+\|P\|_{c b}\right) \gamma\right)^{-1}\right)^{j}$. So from equation (3.3), $\left(x_{j}\right)_{j}$ converges to $y$. As $\pi$ has closed range, it shows that $\pi$ is surjective onto $\mathcal{N}$. To conclude, we just need to apply Proposition 2.4. Clearly,

$$
\max \left\{\|\pi\|_{c b}^{-1},\left\|\operatorname{id}_{\mathcal{M}}\right\|_{c b}^{-1}\right\}=1
$$

and we have seen above that

$$
\left\|\pi-\mathrm{id}_{\mathcal{M}}\right\|_{c b} \leq \varepsilon+\left(1+\|P\|_{c b}\right) \gamma<\|u\|^{-1},
$$

so we may obtain a similarity $S$ such that $\operatorname{id}_{\mathcal{M}}=\operatorname{Ad}_{S} \circ \pi$. As $\mathcal{N}$ is the range of $\pi$, this ends the proof.

Remark 3.10. If one compares the preceding Theorem with Christensen's Theorem 4.1 in [5], the first difference that appears is that, here, we need a near cb-inclusion. But when $\mathcal{M}$ is injective von Neumann algebra, it is known that a near inclusion $\mathcal{M} \subseteq^{\gamma} \mathcal{N}$ implies the near cb-inclusion $\mathcal{M} \subseteq_{c b}^{6 \gamma} \mathcal{N}$ (see Theorem 3.1 in [6], with $D=\mathbb{M}_{n}$ for arbitrary $n$ and $k=1$ thanks to injectivity). Actually, the real difference here is that we assume the $w^{*}$-continuity of the projection $P$, whereas E. Christensen used Tomiyama decomposition of $P$ into normal and singular parts (see [24]). We will discuss a possible analogous decomposition for $\sigma h$-amenable dual operator algebras in the last subsection. Such a decomposition would enable us to get rid of the $w^{*}$-continuity hypothesis on $P$.

The $w^{*}$-continuity of the projection $P$ in the previous theorem is a strong hypothesis. For instance, if $\mathcal{N}$ were selfadjoint, it would be a von Neumann algebra of type I. The next Theorem can be compared with E. Christensen's result Theorem 6.1 in [4]. Note that the following result can be deduced from Theorem 5.3 in [19], but in the type I case, we can improve the bound.

Theorem 3.11. Let $\mathcal{M}, \mathcal{N} \subset \mathbb{B}(H)$ be two unital $w^{*}$-closed operator algebras. We suppose that $\mathcal{N}$ is a von Neumann algebra of type $I$. If $\mathcal{N} \subset^{1} \mathcal{M}$ and $\mathcal{M} \subseteq_{c b}^{\gamma} \mathcal{N}$, with $\gamma<\frac{1}{82}$, then there exists an invertible operator $S$ in the $w^{*}$-closed algebra generated by $\mathcal{M} \cup \mathcal{N}$ such that $S \mathcal{M} S^{-1}=\mathcal{N}$. 
Proof. As $\mathcal{N}$ is a von Neumann algebra of type I, $\mathcal{N}$ is the range of a completely contractive $w^{*}$-continuous projection $P$. As before, let $T=P_{\mid \mathcal{M}}: \mathcal{M} \rightarrow \mathcal{N}$. Now we have the following estimation,

$$
\left\|T-\operatorname{id}_{\mathcal{M}}\right\|_{c b} \leq 2 \gamma, \quad\left\|T^{-1}\right\|_{c b} \leq \frac{1}{1-2 \gamma}
$$

and consequently

$$
\left\|T^{-1}-\operatorname{id}_{\mathcal{N}}\right\|_{c b} \leq\left\|T^{-1}\right\|_{c b}\left\|T-\operatorname{id}_{\mathcal{M}}\right\|_{c b} \leq \frac{2 \gamma}{1-2 \gamma}
$$

and

$$
\left\|\left(T^{-1}\right)^{\vee}\right\|_{c b} \leq\left(2+\left\|T^{-1}\right\|_{c b}\right)\left\|T^{-1}-\mathrm{id}_{\mathcal{N}}\right\|_{c b} \leq \frac{2 \gamma(3-4 \gamma)}{(1-2 \gamma)^{2}} .
$$

The proof essentially follows the previous one, but we want to apply Theorem 3.3 to $T^{-1}$, which is $w^{*}$-continuous (by the Krein-Smulian Theorem) and unital. Here, we put $\mu=(1-2 \gamma)^{-1}, \delta=\frac{2 \gamma(3-4 \gamma)}{(1-2 \gamma)^{2}}$ and

$$
\varepsilon=\frac{8 \gamma(3-4 \gamma)\left((1-2 \gamma)^{2}+4\right)}{(1-2 \gamma)^{4}}
$$

Moreover, as $\mathcal{N}$ is an injective von Neumann algebra, we can find a $\sigma h$-diagonal $u$ of norm 1 (see [8]). Further, one can check that

$$
\delta=\frac{\varepsilon}{4\|u\|+8 \mu^{2}\|u\|^{2}}
$$

so we can apply Theorem 3.3 to $T^{-1}$ and find a unital $w^{*}$-continuous completely bounded homomorphism $\pi: \mathcal{N} \rightarrow \mathcal{M}$ such that $\| T^{-1}-$ $\pi \|_{c b} \leq \varepsilon$. Consequently,

$$
\begin{aligned}
\left\|\pi-\mathrm{id}_{\mathcal{N}}\right\|_{c b} & \leq\left\|\pi-T^{-1}\right\|_{c b}+\left\|T^{-1}-\mathrm{id}_{\mathcal{N}}\right\|_{c b} \\
& \leq \varepsilon+\frac{2 \gamma}{1-2 \gamma}
\end{aligned}
$$

Since $\gamma<\frac{1}{82}$,

$$
\left\|\pi-\mathrm{id}_{\mathcal{N}}\right\|_{c b} \leq \varepsilon+\frac{2 \gamma}{1-2 \gamma}<1
$$

As in the proof of Theorem 3.9, one can prove $\pi$ is injective and has closed range. The surjectivity of $\pi$ comes from the fact for any $y$ in the unit ball of $\mathcal{M}$, there is $x=\pi(T(y))$ in the range of $\pi$ such that

$$
\|y-x\| \leq\left\|T^{-1}-\pi\right\|\|T(y)\| \leq \varepsilon<1 .
$$

hence, by induction as above, we conclude that $\pi$ is bijective. Now the strict inequality in (3.4) enables us to apply Proposition 2.4 to $\pi$ and $\operatorname{id}_{\mathcal{N}}$, which gives the desired similarity. 
The following theorem is a variant of Theorem 5.2 in [19]; now both hypothesis (amenability hypothesis and complementation property) are endowed by the non-selfadjoint algebra.

Theorem 3.12. Let $\mathcal{M}, \mathcal{N} \subset \mathbb{B}(H)$ be two unital $w^{*}$-closed operator algebras. We suppose that $\mathcal{N}$ is a von Neumann algebra and $\mathcal{M}$ is oh-amenable (with $\sigma$ h-diagonal $u$ ) and there is a completely bounded projection $P$ from $\mathbb{B}(H)$ onto $\mathcal{M}$.

If $\mathcal{M} \subset \frac{1}{\|P\|} \mathcal{N}$ and $\mathcal{N} \subseteq_{c b}^{\gamma} \mathcal{M}$ with

$$
\gamma<\frac{1}{\left(1+\|P\|_{c b}\right)\left(3\|u\|+54\|u\|^{2}+243\|u\|^{3}\right)},
$$

then there exists an invertible operator $S$ in the $w^{*}$-closed algebra generated by $\mathcal{M} \cup \mathcal{N}$ such that $S \mathcal{M} S^{-1}=\mathcal{N}$.

Proof. As before we denote $T=P_{\mid \mathcal{N}}$. By Lemma 3.8,

$$
\left\|T-\operatorname{id}_{\mathcal{N}}\right\|_{c b} \leq\left(1+\|P\|_{c b}\right) \gamma<1
$$

and $T$ is bijective and $\left\|T^{-1}\right\|_{c b} \leq \frac{1}{1-\left(1+\|P\|_{c b}\right) \gamma}$. Denote $T^{\mathrm{n}}$ the normal part of $T$. Then we also have

$$
\left\|T^{\mathrm{n}}-\mathrm{id}_{\mathcal{N}}\right\|_{c b} \leq\left(1+\|P\|_{c b}\right) \gamma<1 .
$$

Hence $T^{\mathrm{n}}$ is injective and has closed range. By induction as in Lemma 3.8 , we can prove that $T^{\mathrm{n}}$ is surjective. Let $y$ in the unit ball of $\mathcal{M}$, put $x=T^{-1}(y)$ in $\mathcal{N}$. Then $\left\|y-T^{\mathrm{n}}(x)\right\| \leq\left\|T^{\mathrm{n}}-T\right\|\left\|T^{-1}\right\| \leq \frac{2\left(1+\|P\|_{c b}\right) \gamma}{1-\left(1+\|P\|_{c b}\right) \gamma}$, because

$$
\left\|T^{\mathrm{n}}-T\right\| \leq\left\|T-\mathrm{id}_{\mathcal{N}}\right\|+\left\|T^{\mathrm{n}}-\mathrm{id}_{\mathcal{N}}\right\| \leq 2\left(1+\|P\|_{c b}\right) \gamma .
$$

Therefore $T^{\mathrm{n}}$ is a linear $w^{*}$-continuous cb-isomorphism from $\mathcal{N}$ onto $\mathcal{M}$. Define $V=\left(T^{\mathrm{n}}\right)^{-1}$ which is also a $w^{*}$-continuous (by Krein-Smulyan Theorem) cb-isomorphism from $\mathcal{M}$ onto $\mathcal{N}$.

Now the proof essentially follows the proof of Theorem 3.2 in [19]. To apply Theorem 3.3, we need to unitize $V$. Note that $\|V(1)-1\| \leq$ $\frac{\left(1+\|P\|_{c b}\right) \gamma}{1-\left(1+\|P\|_{c b}\right) \gamma}<1$, so $V(1)$ is invertible in $\mathcal{N}$ and we obtain

$$
\left\|V(1)^{-1}\right\| \leq \frac{1}{1-\|V(1)-1\|} \leq \frac{1-\left(1+\|P\|_{c b}\right) \gamma}{1-2\left(1+\|P\|_{c b}\right) \gamma}
$$

Denote $L=V(1)^{-1} V$, so $L$ is a unital $w^{*}$-continuous completely bounded isomorphism from $\mathcal{M}$ onto $\mathcal{N}$ and

$$
\|L\|_{c b} \leq\left\|V(1)^{-1}\right\|\|V\|_{c b} \leq\left\|V(1)^{-1}\right\|\left\|T^{-1}\right\|_{c b} \leq \frac{1}{1-2\left(1+\|P\|_{c b}\right) \gamma} .
$$


Fix $n$, let $x$ in unit ball of $\mathbb{M}_{n}(\mathcal{N})$, from Equation (3.5) we have

$$
\begin{aligned}
\left\|L_{n}(x)-x\right\| & \leq\left\|I_{n} \otimes V(1)^{-1}\left(V_{n}(x)-x\right)\right\|+\left\|I_{n} \otimes V(1)^{-1} x-x\right\| \\
& \leq\left\|V(1)^{-1}\right\|\left\|V-\operatorname{id}_{\mathcal{N}}\right\|_{c b}+\left\|V(1)^{-1}\right\|\|V(1)-1\| \\
& \leq \frac{2\left(1+\|P\|_{c b}\right) \gamma}{1-2\left(1+\|P\|_{c b}\right) \gamma}
\end{aligned}
$$

so

$$
\left\|L-\operatorname{id}_{\mathcal{N}}\right\|_{c b} \leq \frac{2\left(1+\|P\|_{c b}\right) \gamma}{1-2\left(1+\|P\|_{c b}\right) \gamma} .
$$

Therefore, by Lemma 3.8 and Equation (3.6) we estimate the defect of multiplicativity of $L$.

$$
\left\|L^{\vee}\right\|_{c b} \leq\left(2+\|L\|_{c b}\right)\left\|L-\mathrm{id}_{\mathcal{N}}\right\|_{c b} \leq \frac{6\left(1+\|P\|_{c b}\right) \gamma}{\left(1-2\left(1+\|P\|_{c b}\right) \gamma\right)^{2}} .
$$

We can apply Theorem 3.3 to L. Put

$$
\mu=\frac{1}{1-2\left(1+\|P\|_{c b}\right) \gamma} \text { and } \delta=\frac{6\left(1+\|P\|_{c b}\right) \gamma}{\left(1-2\left(1+\|P\|_{c b}\right) \gamma\right)^{2}}
$$

Let

$$
\varepsilon=\delta\left(4\|u\|+8 \mu^{2}\|u\|^{2}\right)=\frac{6\left(1+\|P\|_{c b}\right) \gamma}{\left(1-2\left(1+\|P\|_{c b}\right) \gamma\right)^{2}}\left(4+\frac{8}{(1-4 \gamma)^{2}}\right) .
$$

By Theorem 3.3 we can find a unital $w^{*}$-continuous completely bounded homomorphism $\pi: \mathcal{M} \rightarrow \mathcal{N}$ such that

$$
\|L-\pi\|_{c b} \leq \varepsilon
$$

Consequently, from Equation (3.7)

$$
\begin{aligned}
\left\|\pi-\mathrm{id}_{\mathcal{N}}\right\|_{c b} & \leq\|\pi-L\|_{c b}+\left\|L-\mathrm{id}_{\mathcal{N}}\right\|_{c b} \\
& \leq \varepsilon+\frac{2\left(1+\|P\|_{c b}\right) \gamma}{1-2\left(1+\|P\|_{c b}\right) \gamma}<1
\end{aligned}
$$

because $\gamma<\frac{1}{\left(1+\|P\|_{c b}\right)\left(3\|u\|+54\|u\|^{2}+243\|u\|^{3}\right)}$. Hence, applying Proposition 2.4 to $\pi$ and $\operatorname{id}_{\mathcal{N}}$, we find an invertible operator $S$ in the $w^{*}$-closed algebra generated by $\mathcal{M} \cup \mathcal{N}$ such that

$$
\operatorname{Ad}_{S} \circ \pi=\mathrm{id}_{\mathcal{N}}
$$

To achieve the proof, it is sufficient to prove that the range of $\pi$ is $\mathcal{M}$. Note from the previous equation that $\pi$ is injective and has closed range. Let $y$ is in the unit ball of $\mathcal{M}$, then

$$
\left\|\pi\left(L^{-1}(y)\right)-y\right\| \leq\|\pi-L\|_{c b}\left\|L^{-1}\right\|_{c b} .
$$


To prove the surjectivity (by induction as in Lemma 3.8) we just need to check that $\|\pi-L\|_{c b}\left\|L^{-1}\right\|_{c b}$ is strictly smaller than one. However, from Equation (3.7)

$$
\left\|L^{-1}\right\|_{c b} \leq \frac{1}{1-\left\|L-\mathrm{id}_{\mathcal{N}}\right\|_{c b}} \leq \frac{1-2\left(1+\|P\|_{c b}\right) \gamma}{1-4\left(1+\|P\|_{c b}\right) \gamma}
$$

it follows that

$$
\|\pi-L\|_{c b}\left\|L^{-1}\right\|_{c b} \leq \frac{1-2\left(1+\|P\|_{c b}\right) \gamma}{1-4\left(1+\|P\|_{c b}\right) \gamma} \varepsilon
$$

which is strictly smaller than 1 .

3.3. Predual properties: a special case. In this short subsection, we prove Tomiyama decomposition for a special subclass of $\sigma h$-amenable. Here we deal with a dual operator algebra $\mathcal{M}$ admitting a virtual $h$ diagonal (in the sense of [2]), it means that $\mathcal{M}$ admits an element $u \in\left(\mathcal{M} \otimes_{h} \mathcal{M}\right)^{* *}$ satisfying conditions $(C 1)$ and $(C 2)$. We recall that the normal Haagerup tensor product of dual operator spaces can defined by $\mathcal{X} \otimes_{\sigma h} \mathcal{Y}=\left(\left(\mathcal{X} \otimes_{h} \mathcal{Y}\right)_{\sigma}^{*}\right)^{*}$, where $\left(\mathcal{X} \otimes_{h} \mathcal{Y}\right)_{\sigma}^{*} \subset\left(\mathcal{X} \otimes_{h} \mathcal{Y}\right)^{*}$ denotes the subspace of all completely bounded bilinear forms which are separately $w^{*}$-continuous (see Paragraph 1.6.8 of [2]). By duality, this last inclusion induces a map from $\left(\mathcal{M} \otimes_{h} \mathcal{M}\right)^{* *}$ onto $\mathcal{M} \otimes_{\sigma h} \mathcal{M}$ and this map sends virtual $h$-diagonals inside normal virtual $h$-diagonals.

Proposition 3.13. Let $\mathcal{M} \subset \mathbb{B}(H)$ be a unital $w^{*}$-closed operator algebra, denote $\mathcal{M}_{*}$ its standard predual. Suppose that $\mathcal{M}$ admits a diagonal $u \in\left(\mathcal{M} \otimes_{h} \mathcal{M}\right)^{* *}$.

(1) There is a unique central idempotent $p \in \mathcal{M}^{* *}$ such that $R_{p}\left(\mathcal{M}^{*}\right)=$ $\mathcal{M}_{*}$ (see Prop. 1.17.7 in [22] for notation). Moreover, $\|p\| \leq$ $\|u\|$.

(2) For any bounded linear map $T: \mathcal{M} \rightarrow \mathcal{X}$ into a dual Banach space $\mathcal{X}=\left(\mathcal{X}_{*}\right)^{*}, T$ can be decomposed into $w^{*}$-continuous and 'singular' part.

Proof. By paragraph 7.4.9 in [2], $u$ is actually a normal virtual $h$ diagonal for the dual operator algebra $\mathcal{M}^{* *}$, in particular it enjoys the $w^{*}$-complementation property. As in [22], let $\mathcal{M}_{*}^{0} \subset \mathcal{M}^{* *}$ be the polar of $\mathcal{M}_{*}$, then $\mathcal{M}_{*}^{0}$ is a $w^{*}$-closed ideal of $\mathcal{M}^{* *}$, hence its annihilator is also an $w^{*}$-closed ideal of $\mathcal{M}^{* *}$. By proposition 2.8 above, there exists a unique central idempotent $p \in \mathcal{M}^{* *}$ such that $\mathcal{M}_{*}^{0}=(1-p) \mathcal{M}^{* *}$. The rest follows [24], one just has to define the $w^{*}$-continuous part, for $y \in \mathcal{M}$ by

$$
T^{n}(y)=\left(\left(T^{*}\right) \mathcal{X}_{*}\right)^{*}(p y)
$$


and the singular part by

$$
T^{s}(y)=\left(\left(T^{*}\right) \mid \mathcal{X}_{*}\right)^{*}((1-p) y) .
$$

Question. Can we find an analogous Tomiyama decomposition for $\sigma h$-amenable dual operator algebras ? More generally, it would be beneficial to investigate the predual properties of $\sigma h$-amenable dual operator algebras.

Acknowledgements. The author is grateful to Narutaka Ozawa for hosting him at RIMS in Kyoto.

\section{REFERENCES}

[1] D.P. Blecher, U. Kashyap, Morita equivalence of dual operator algebras. J. Pure Appl. Algebra 212 (2008), no. 11, 2401-2412.

[2] D.P. Blecher, C. Le Merdy, Operator algebras and their modules - an operator space approach. London Mathematical Society Monographs. New Series, 30. Oxford Science Publications.

[3] D.P. Blecher, B. Magajna, Duality and operator algebras: automatic weak* continuity and applications. J. Funct. Anal. 224 (2005), no. 2, 386-407.

[4] E. Christensen, Perturbations of type I von Neumann algebras. J. London Math. Soc. (2) 9 (1974/75), 395-405.

[5] E. Christensen, Perturbations of operator algebras. Invent. Math. 43 (1977), no. $1,1-13$.

[6] E. Christensen, Near inclusions of $C^{*}$-algebras. Acta Math. 144 (1980), no. 3-4, 249-265.

[7] K.R. Davidson, Perturbations of reflexive operator algebras. J. Operator Theory 15 (1986), no. 2, 289-305.

[8] E.G. Effros, Amenability and virtual diagonals for von Neumann algebras. J. Funct. Anal. 78 (1988), no. 1, 137-153.

[9] E.G. Effros, Z.J. Ruan Operator spaces. London Mathematical Society Monographs. New Series, 23. The Clarendon Press, Oxford University Press, New York, 2000.

[10] J. A. Gifford, Operator algebras with a reduction property, PhD Dissertation, Australian National University, 1997.

[11] K. Jarosz, Ultraproduct and small bound perturbations. Pacific Journal of Math., 148 (1991), no. 1, 81-88.

[12] B.E. Johnson, Perturbations of Banach algebras. Proc. London Math. Soc. (3) 34 (1977), no. 3, 439-458.

[13] E.C. Lance, Cohomology and perturbations of nest algebras. Proc. London Math. Soc. (3) 43 (1981), no. 2, 334-356.

[14] V.I. Paulsen, Vern Completely bounded maps and operator algebras. Cambridge Studies in Advanced Mathematics, 78. Cambridge University Press, Cambridge, 2002.

[15] V.I. Paulsen, R.R. Smith, Diagonals in tensor products of operator algebras. Proc. Edinb. Math. Soc. (2) 45 (2002), no. 3, 647-652. 
[16] G. Pisier, Introduction to operator space theory. London Mathematical Society Lecture Note Series, 294. Cambridge University Press, Cambridge, 2003.

[17] D.R. Pitts, Close CSL algebras are similar. Math. Ann. 300 (1994), no. 1, $149-156$

[18] I. Raeburn, J.L. Taylor, Hochschild cohomology and perturbations of Banach algebras. J. Functional Analysis 25 (1977), no. 3, 258-266.

[19] J. Roydor, Dual operator algebras close to injective von Neumann algebras. ArXiv.

[20] V. Runde, Lectures on amenability. Lecture Notes in Mathematics, 1774. Springer-Verlag, Berlin, 2002.

[21] V. Runde, A Connes-amenable, dual Banach algebra need not have a normal, virtual diagonal. Trans. Amer. Math. Soc. 358 (2006), no. 1, 391-402.

[22] S. Sakai, $C^{*}$-algebras and $W^{*}$-algebras. Ergebnisse der Mathematik und ihrer Grenzgebiete, Band 60. Springer-Verlag, New York-Heidelberg, 1971.

[23] A.M. Sinclair, R.R. Smith, Hochschild cohomology of von Neumann algebras. London Mathematical Society Lecture Note Series, 203. Cambridge University Press, Cambridge, 1995.

[24] J. Tomiyama, On the projection of norm one in $W^{*}$-algebras. III. Tôhoku Math. J. (2) 111959 125-129.

Jean Roydor, Department of Mathematical Sciences, Tokyo, 1538914, Japan.

roydor@ms.u-tokyo.ac.jp

\section{Current Address:}

Jean Roydor, Institut de Mathmatiques de Bordeaux, Universit Bordeaux 1 351, cours de la Libration - F 33405 TALENCE cedex, France

jean.roydor@math.u-bordeaux1.fr 